\title{
Correlation between the sagittal plane in adults and the automatic postural system: a longitudinal study with a $\mathbf{2 4}$ month follow-up period
}

\section{A Fimiani}

Address: via Dello Stadio 79 Ischia (NA), 80077, Italy

Email: A Fimiani - fimianiantonio@libero.it

from 6th International Conference on Conservative Management of Spinal Deformities

Lyon, France. 21-23 May 2009

Published: 14 December 2009

Scoliosis 2009, 4(Suppl 2):O27 doi:I0.I I86/I748-7I6I-4-S2-O27

This abstract is available from: http://www.scoliosisjournal.com/content/4/S2/O27

(C) 2009 Fimiani; licensee BioMed Central Ltd.

\section{Objectives}

The Sagittal Plane is governed by the correct balance of the pelvis. The muscle chains are governed by the exoreceptors. Therefore, newly corrected exoreceptor information brings about a subsequent realignment of the musculoskeletal axis.

\section{Background}

Since the early 1980's a cybernetic concept of governing balance was introduced. In 2005 J. C. De Mauroy, introduced the existence of a strange attractor, and in 2008, A. Fimiani hypothesized the role of the pelvis as the strange attractor of the postural system.

\section{Materials and methods}

The research subjects were made up of 68 adults between the ages of 18 and 70, of which 48 were female and 20 were male. The patients underwent only receptor treatment, following the techniques by B. Bricot and by Bourdiol. All patients were checked with x-rays and photographic evidence, measured on the dynamometric platform, and tested with the S. F. McGill Questionnaire. The time of observation was 24 months.

\section{Results}

Comparing photographs of the patients' backs showed that after 24 months all the patients adopted a new posture. From the x-rays it was possible to note only lumbar and dorsal curvature. Two subgroups of patients were individually characterized by age. Group A consisted of 22 people between 40 and 70 years old. Group B consisted of 30 people between 18 and 30 years old. In group A, both the lumbar curvature $(\mathrm{p}=0.38)$ and dorsal curvature $(\mathrm{p}=$ 0.067 ) do not show any significant variation between them. In group $\mathrm{B}$, both lumbar curvature $(\mathrm{p}=0.0349)$ and dorsal curvature $(\mathrm{p}=0.0484)$ show significant variation. An evaluation of lumbar pain demonstrated a significant statistical improvement ( $\mathrm{p}=<0.000001)$.

\section{Conclusion}

The fundamental problems for the patient are represented by pain and aesthetic deformity. In both cases, observation shows that patients experienced and obtained a significant improvement. Analysis of the x-rays points out that if the skeleton isn't compromised in any way, it modifies itself following the new muscular equilibrium, even in adults. So we can conclude that correct external stimulation of the automatic postural system influences the position of the body in relation to its surrounding space. 